\title{
Self-Assembled Large-Scale Monolayer of Au Nanoparticles at Air/Water Interface Used as a SERS Substrate
}

\author{
Qinghua Guo, Minmin Xu, Yaxian Yuan*, Renao Gu, and Jianlin Yao*
}

College of Chemistry, Chemical Engineering and Materials Science, Soochow University, Suzhou 215123, China

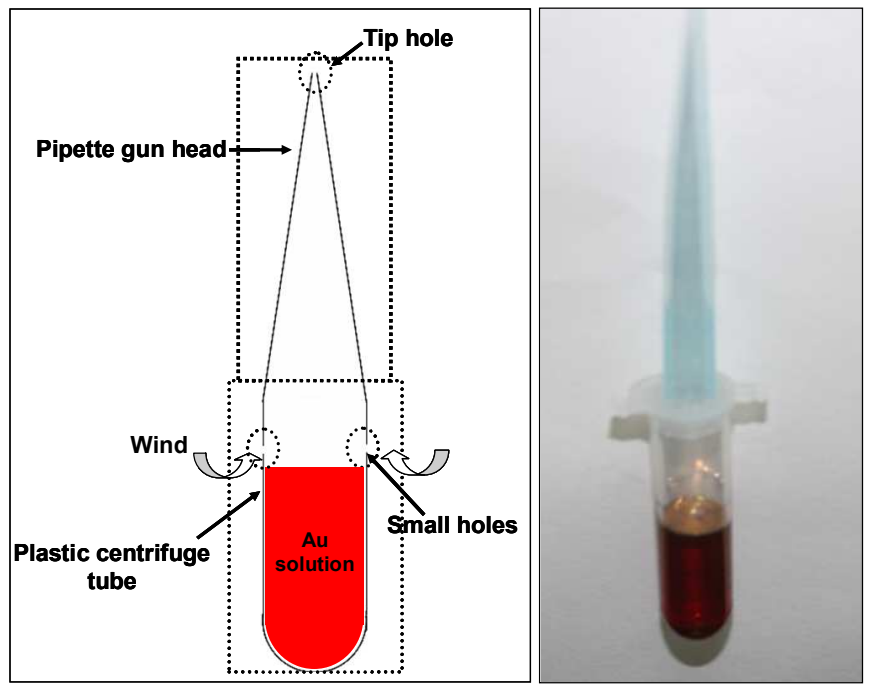

Figure S1 Schematic diagram of the lab made chimney device for controlling the evaporation processes to fabricate Au NPs monolayer film. 


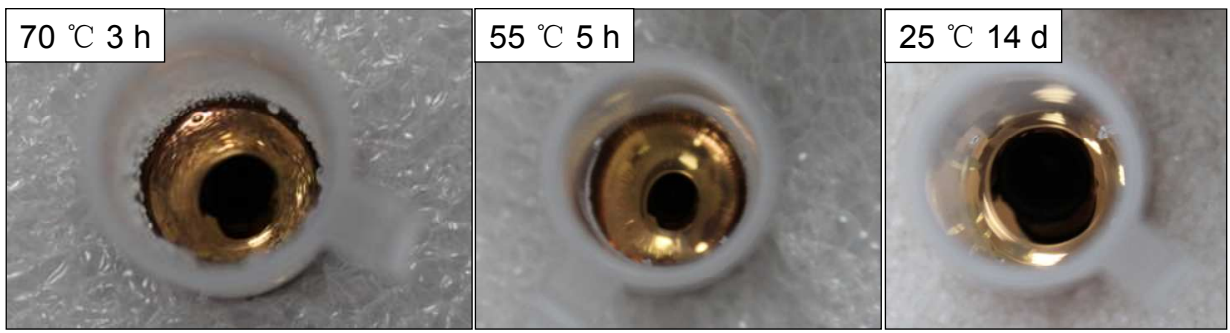

Figure S2 Formation process of $\mathrm{Au}$ monolayer film revealed that the higher evaporation temperature employed, the shorter assembly time needed

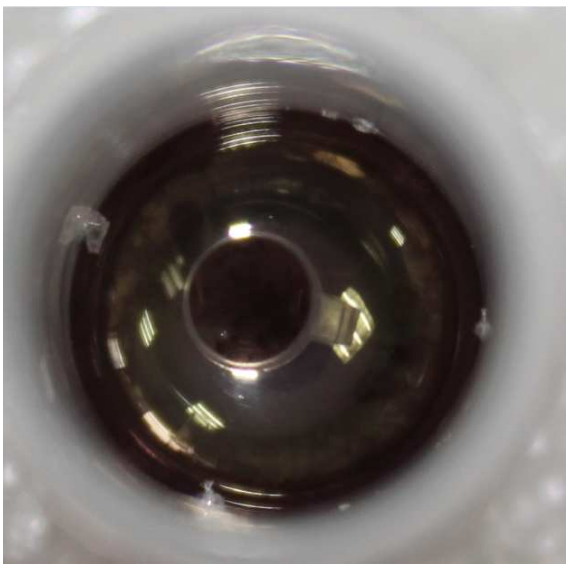

Figure S3 Optic photo of $45 \mathrm{~nm}$ Au NPs solution (Frens' way) several days after the addition of PVP. 


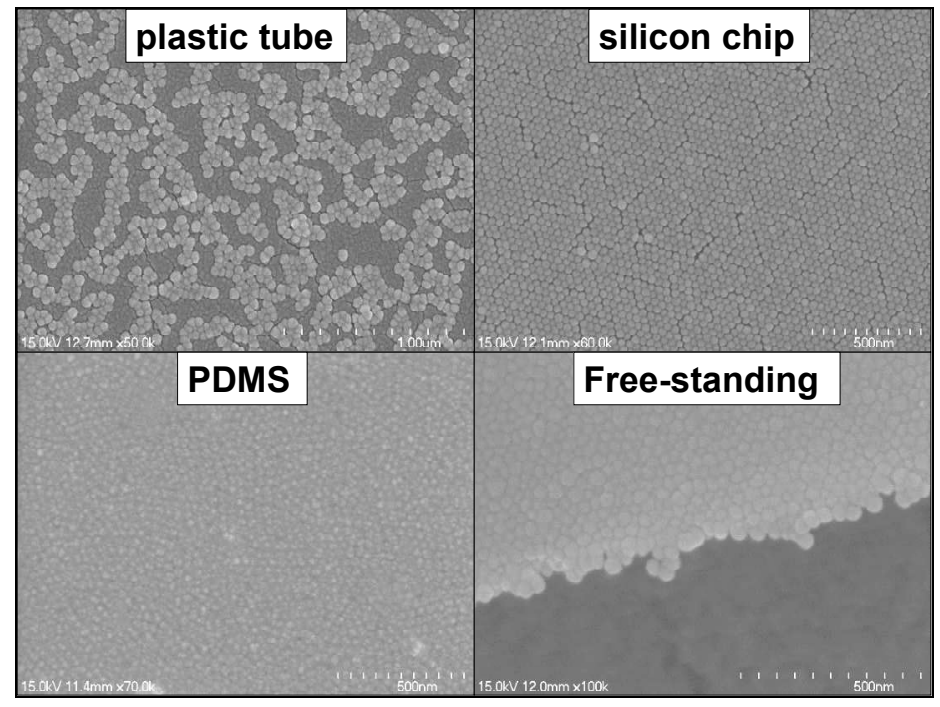

Figure S4 the monolayer film was transferred to different kinds of solid surfaces 


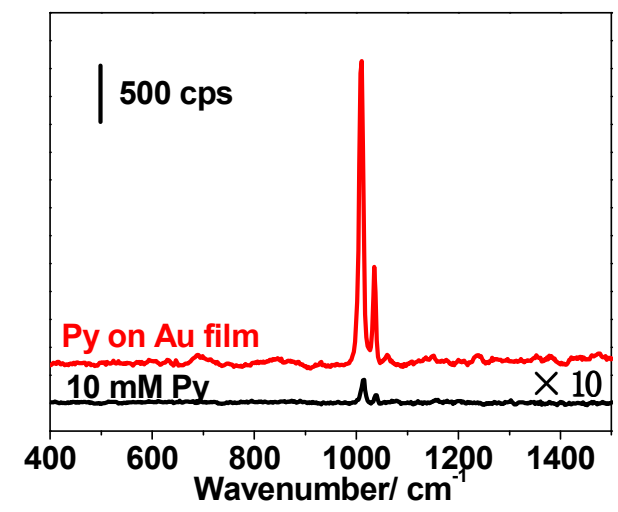

Figure S5 Raman spectrum of $10 \mathrm{mM}$ Py and SERS spectrum of $1 \mathrm{mM}$ Py adsorbed on $\mathrm{Au}$ monolayer film (under the same condition)

Pyridine (Py) was chosen as the model molecular to calculate the SERS EFs of Au monolayer film. Raman spectrum of $10 \mathrm{mM} \mathrm{Py}_{(\mathrm{aq})}$ (black) and SERS spectrum of Py absorbed on Au monolayer film (red) were shown in Figure S5. Au film was fully covered by a monolayer of Py, whose molecular footprint was about $0.25 \mathrm{~nm}^{2}$. This assumption represented the theoretically maximum number of molecules and was therefore an overestimate. Thus, the calculated EFs would likely be an underestimate rather than an overestimate of the enhancement. We employed the peak at 1002 $\mathrm{cm}^{-1}$ to estimate the EF through the following equation:

$$
E F=\frac{I_{\text {SERS }}}{N_{\text {SERS }}} / \frac{I_{\text {bulk }}}{N_{\text {bulk }}}
$$

where $\mathrm{I}_{\mathrm{SERS}}$ and $\mathrm{I}_{\text {bulk }}$ were the intensities of the same band for the SERS and bulk spectra, $\mathrm{N}_{\text {bulk }}$ was the number of molecules probed for a bulk sample, and $\mathrm{N}_{\mathrm{SERS}}$ was the number of molecules probed in SERS. The calculated EF of Au monolayer film was about $5 \times 10^{6}$. Actually, the existence of PVP would effect the adsorption of Py, so the real SERS EF of Au monolayer film was low estimated. 